\title{
La epidemiología en busca de contexto social
}

\section{Epidemiology in search of social context}

\author{
Octavio Martínez • Bogotá, D.C. (Colombia)
}

\begin{abstract}
Resumen
A la epidemiología de los factores de riesgo con la individualidad de sus mediciones en un vacío social, se le endilga el fracaso de las estrategias preventivas para modificar prácticas insalubres individuales y comunitarias, dado lo restrictivo de su abordaje, circunscrito a factores de riesgo "objetivos" y fuertes, que no considera variables de grupo y que estima como marginales para la investigación epidemiológica la pléyade de determinantes historicosociales, económicos, políticos, culturales y de género que influencian la salud de las poblaciones. A esta epidemiología con orientación biomédica, cercana a la medicina de hospital centrada en procedimientos de laboratorio biológicos, moleculares y fisicoquímicos, y en sinergia con posturas derivadas del individualismo de los estilos de vida, se la confronta con alternativas teóricas epidemiológicas con pretensiones de ser más comprensivas y sistémicas, capaces de abordar explicaciones causales y de patrones de distribución de salud y enfermedad en la población desde la multidimensionalidad de determinantes sociales, psicológicos, políticos, económicos y culturales, sobre los cuales se actúa para evitar y corregir inequidades sociales en salud. (Acta Med Colomb 2012; 37: 93-96)
\end{abstract}

Palabras clave: factores de riesgo, determinantes sociales, epidemiología.

\begin{abstract}
Failure of preventive strategies to modify unhealthy individual and community practices has been attributed to the epidemiology of risk factors with the individuality of its measurements in a social vacuum, given its restrictive approach, circumscribed to "objective" and strong risk factors that don't consider group variables and that estimates as marginal for epidemiological investigation the generation of historic, social, economic, political, cultural and gender determinants which influence the health of populations.

This epidemiology with biomedical orientation, close to hospitalary medicine centered in biological, molecular and physicochemical laboratory procedures, and in synergy with attitudes derived from individualism of lifestyles, is confronted with theoretical epidemiological alternatives pretending to be more comprehensive and systemic, able to come up to causal explanations and of health distribution patterns and disease in the population from the multidimensionality of social, psychological, political, economic and cultural determinants, on which we must act to avoid and correct social health inequities. (Acta Med Colomb 2012; 37: 93-96)
\end{abstract}

Keywords: risk factors, social determinants, epidemiology.
Dr. Octavio Martínez Betancur: Profesor Asociado - Departamento de Medicina Interna, Facultad de Medicina, Universidad Nacional de Colombia. Bogotá, D.C. (Colombia).

Correspondencia: Dr. Octavio Martínez. E-mail: omartinezb@unal.edu.co

Recibido: 18/XI/2011 Aceptado: 22/II/2012

\section{De la vocación social de la epidemiología}

Los escritos sobre las condiciones sociales y de salud de las clases trabajadoras realizados en Francia por Louis René Villermé (1), en Alemania por Rudolf Virchow (2-4) y en Inglaterra por Friedrich Engels (5), prefiguran investigaciones epidemiológicas en ausencia de una teoría formal, con vocación de identificar los determinantes sociales de salud y enfermedad en la población, particularmente pobreza y privaciones material, cultural y política, productos del gradiente de poder de la estructura social (6). Una enfermedad se hace socialmente significativa no únicamente por su frecuencia de presentación sino también por su patrón de ocurrencia determinado por las condiciones sociales prevalentes, lo que explica la frecuencia diferencial de enfermedades entre poblaciones y entre grupos sociales de una misma población $(7,8)$. O bien, la distribución de salud y enfermedad en una sociedad refleja la distribución de bienestar y desventajas sociales en dicha colectividad (9).

Las reformas sociales y sanitarias en Europa durante la segunda mitad del siglo XIX, los descubrimientos de los microorganismos causantes de enfermedad y el control de su transmisión, testificaron el cambio histórico en el objeto de 
estudio epidemiológico, de enfermedades infecciosas agudas y epidémicas a enfermedades crónicas no infecciosas, y la transición de la epidemiología premoderna a la epidemiología moderna, que rinde mayor importancia al avance en los diseños de investigación y a los métodos cuantitativos analíticos, sin mayor preocupación por el desarrollo conceptual de la epidemiología, nada diferente al enfoque positivista de adecuar herramientas metodológicas para el estudio de causalidad sobre datos individuales (10-12).

A partir de 1960 se suceden en el mundo movimientos populares que retan los poderes coloniales, el poder del capital y la represión de los gobiernos militares y totalitarios, se enjuicia críticamente la ciencia encastillada en el positivismo popperiano, y se inician esfuerzos para integrar la epidemiología con teorías social, económica y política prestadas de las ciencias sociales. A partir de este cruce disciplinar, los determinantes sociales que afectan la salud y la enfermedad de las poblaciones dejan de ser variables demográficas marginales en la investigación epidemiológica, para convertirse en causas de las causas de enfermedad o riesgos exógenos patogénicos por derecho propio $(8,9,13,14)$.

\section{La epidemiología en busca de contexto social}

El paradigma de la "caja negra" en epidemiología vincula la exposición con la enfermedad, asociación fortuita que se convierte en un vínculo causal sin explicación lógica puesto que el presunto mecanismo causal, no obstante estar implícitamente presente en el interior de la "caja", permanece desconocido o no es evidente ("negra") (15). Este paradigma de corte determinista que sustenta la epidemiología de los factores de riesgo, fiel a la concepción de dar prioridad a lo medicobiológico en el estudio de variaciones interindividuales del riesgo de enfermedad, controla como confundidores a los factores corriente arriba de la asociación causal estudiada y a los factores de dominios no clínicos que inciden sobre la evolución del nivel individual (16-19). Esta epidemiología académica asume que las poblaciones son solamente agregados de individuos quienes eligen libre y responsablemente, comportamientos y hábitos personales, y que estudios locales con individuos seleccionados arbitrariamente, metodológicamente bien realizados pero sin contexto social a su alrededor, pueden estimar presuntas relaciones de riesgo individual con carácter universal $(20$, 21). Los individuos deben aceptar la culpa por muchos de los efectos deletéreos sobre su salud como consecuencia de sus estilos de vida y, al no reconocer el contexto sociohistórico del colectivo como el principal determinante del nivel absoluto del riesgo, absuelve de responsabilidades a la sociedad (22-25).

Los resultados derivados de los estudios tradicionales de los factores de riesgo a nivel individual en ausencia de la perspectiva sociohistórica del colectivo, han sido marginalmente fértiles en apoyar la función preventiva de la salud pública (26). A la epidemiología de los factores de riesgo con la individualidad de sus mediciones en un vacío social, se le pregunta por la postergación de explicaciones de lo que sucede en el interior de la "caja" en el estudio de causalidad, y se le endilga el fracaso de las estrategias preventivas para modificar prácticas insalubres individuales y comunitarias, dado lo restrictivo de su abordaje, circunscrito a factores de riesgo "objetivos" y fuertes, que no considera variables de grupo y desestima el valor de la pléyade de determinantes historicosociales, económicos, políticos, culturales y de género que influencian la salud de las poblaciones. Los métodos analíticos de la caja negra que conceptualizan el riesgo como un fenómeno de base individual, no pueden capturar el riesgo que está determinado por cómo los individuos están conectados. Cada población tiene su propia historia, cultura, organización y divisiones económicas y sociales, las cuales influencian cómo y por qué las personas están expuestas a factores particulares (27-30).

Algunos sectores académicos en el campo de la epidemiología de factores de riesgo para enfermedad cardiovascular, critican la búsqueda "circular" con leves refinamientos de los mismos factores de riesgo "añosos", ejemplo extremo de ratificación de la consistencia de asociaciones. Esta epidemiología circular es definida como la continuación de tipos específicos de estudios epidemiológicos más allá del punto de duda razonable de la verdadera existencia o ausencia de una asociación importante, que paraliza la evolución de estudios epidemiológicos (31).

Otras críticas se dirigen a las inferencias unas veces asertivas y seguidamente contradictorias sobre comparaciones entre conjuntos de múltiples factores de riesgo relacionados con estilos de vida y desenlaces en salud, inferencias basadas en efectos de pequeño tamaño obtenidos de estudios epidemiológicos observacionales de enfermedades crónicas. Se habla de la crisis de credibilidad de la epidemiología de riesgos pequeños, resultados explicados en parte por azar, confusión y sesgos en los estudios, cuya solución se entrevé posible con mediciones más sensibles de exposiciones y desenlaces, tras el acople de la epidemiología con la genética molecular en un marco conceptual evolucionista (32-34). Genómica y evolución, parecen no corregir la deriva de la epidemiología de enfermedades crónicas y de riesgos pequeños; esta nueva área de estudio sólo estructura mayores maquinaciones metodológicas y estadísticas, con una maraña más densa en el interior de la "caja negra", sin relevancia para la salud pública y, en medicina clínica, sólo información "tentativa", inestable y huérfana de validaciones (35-42).

Con la fascinación económica que auspicia la epidemiología adscrita a la industria farmacéutica, las prioridades actuales han dirigido las preguntas hacia las interacciones geneticoambientales y moleculares del riesgo individual de enfermedad, sin involucrar las fuerzas sociales determinantes de la salud de las poblaciones (43). Puede ser atractivo reconocer un paradigma genético o molecular que domine la epidemiología actual, no menos que en su tiempo fue el caso de la teoría del germen para el paradigma infeccioso, 
con la idea de causalidad específica y reducción nuevamente de la epidemiología a una ciencia de laboratorio, no aunada a la salud pública sino a la industria, a sus implicaciones mercantiles y a una nostalgia eugenésica $(12,44-50)$.

Son las teorías explicativas epidemiológicas sobre dominios causales específicos y no las metodologías y herramientas empleadas, las que rigen científicamente la investigación epidemiológica. La enseñanza fundadora de la teoría epidemiológica establece que el sujeto de la investigación es la población en su contexto social. Para explicar el patrón de producción social de enfermedad, la teoría organiza y explicita el cuerpo de conocimientos en interrelaciones coherentes que permiten entender por qué algunas poblaciones se enferman más que otras. Explicar la distribución de enfermedad no es lo mismo que explicar el mecanismo de enfermedad. Teorías de la distribución de enfermedad en la población no son las mismas teorías de causa individual de enfermedad. Por tanto, es clave que las teorías epidemiológicas de distribución poblacional de enfermedad evalúen si los mecanismos de enfermedad postulados son compatibles o no con los patrones espaciotemporales y sociales de distribución de enfermedad $(24,51)$.

La epidemiología no se restringe a métodos ni herramientas para obtener y analizar datos sobre enfermedades en poblaciones humanas. El fraccionamiento del quehacer epidemiológico, derivado de las herramientas empleadas para definir factores de riesgo y factores protectores individuales de enfermar, aleja la epidemiología de su función originaria de generar conocimiento científico necesario para la formulación de políticas públicas encaminadas a mejorar la salud de las comunidades, prevenir enfermedades y sufrimiento innecesario, y eliminar las inequidades en salud (52-56). La epidemiología de red causal que entiende las causas de enfermedad como el modelamiento de relaciones complejas entre múltiples factores individuales de riesgo de enfermar, biomédicos y de comportamiento individual, debe cobijarse bajo una epidemiología que analice los sistemas sociales que generan patrones de enfermedad en las poblaciones. Por supuesto, los estudios epidemiológicos en las poblaciones involucran individuos que tienen exposiciones específicas en todos los niveles (poblacional, individual, micro nivel), pero la distinción importante es si se conceptualiza el marco de los determinantes y de la distribución de enfermedad a nivel del colectivo y si las exposiciones se contextualizan social e históricamente. Si la perspectiva sociohistórica del proceso salud-enfermedad del colectivo se pierde, la epidemiología se convierte en una herramienta de medición para probar hipótesis, una "factoría de riesgos" inconsistentes y pequeños $(16,57-60)$.

A esta epidemiología con orientación biomédica, cercana a la medicina de hospital centrada en procedimientos de laboratorio biológicos, moleculares y fisicoquímicos, y en sinergia con posturas derivadas del individualismo de los estilos de vida, se la confronta con alternativas teóricas epidemiológicas con pretensiones de ser más comprensivas y sistémicas, capaces de abordar explicaciones causales y de patrones de distribución de enfermedad en la población desde la multidimensionalidad de determinantes sociales, psicológicos, políticos, económicos y culturales, sobre los cuales se actúa para evitar y corregir inequidades en salud. Las perspectivas teóricas epidemiológicas sociopolítica y psicosocial, consideran que los determinantes tanto sociales como politicoeconómicos estructurales ("societales") corriente arriba del desenlace en salud, deben identificarse y estudiarse no como confundidores sino como causas de las causas de desigualdad e inequidad en salud. La vertiente epidemiológica ecosocial habla de múltiples niveles de organización más allá de lo individual (paradigma de las "cajas chinas"), multiniveles que impactan el proceso saludenfermedad-atención, y que ameritan ser evaluados en la complejidad de sus interacciones para explicar qué y quién direcciona los cambios en los patrones de distribución de enfermedad e inequidad social en salud (16, 61-65).

Más que simplemente adicionarle a la biología el análisis social, o sumarle "factores sociales" al análisis biológico, las epidemiologías sociales proponen una aproximación integradora sistémica capaz de generar nuevas hipótesis, y no simplemente reinterpretar factores identificados por una aproximación (biológica) en términos de otras (social y "societal"). Los retos para la epidemiología emergente son, la construcción teórica que unifique perspectivas y el ajuste metodológico para aproximarse a dinámicas no lineales entre las diferentes fuerzas de organización social que impactan la evolución del nivel individual, y desarrollar la perspectiva ética que guíe las acciones políticas para el cambio de los patrones de inequidad social en salud $(16,65)$.

\section{Referencias}

1. Morabia A. Epidemiological methods and concepts in the nineteenth century and their influences on the twentieth century. In: Holland WW, Olsen J, Florey C. The development of modern epidemiology. Personal report from those who were there. Oxford: Oxford University Press; 2007: 17-29.

2. Virchow R (Archiv. Für Patholog. Anatomie u. Physiologie u. für klin. Medicin, 1848, Vol. II, Nos. 1 and 2). Report on the Typhus Epidemic in Upper Silesia. Social Medicine 2006; 1: 11-98.

3. Taylor R, Rieger A. Rudolf Virchow on the typhus epidemic in Upper Silesia: an introduction and translation. Sociol Health Illness 1984; 6: 201-217.

4. Azar HA. Rudolf Virchow, not just a pathologist: a re-examination of the report on the Typhus Epidemic in Upper Silesia. Ann Diagn Pathol 1997; 1: 65-71.

5. Engels F. La situación de la clase obrera en Inglaterra. Moscú: Progreso; 1980.

6. Williams GH. The determinants of health: structure, context and agency. Sociol Health Illness 2003; 25: 131-154.

7. Rosen G. Approaches to a concept of social medicine. A historical survey. Milbank $Q$ 1948; 26: 7-21.

8. Cassel J. Social science theory as a source of hypotheses in epidemiological research. Am J Public Health 1964; 54: 1482-1488.

9. Honjo K. Social epidemiology: definition, history, and research examples. Env Health \& Prev Med 2004; 9: 193-199.

10. Susser M. Epidemiology today: 'a thought-tormented world'. Intern J Epidemiol 1989; 18: 481-488.

11. Bophal R. Paradigms in epidemiology textbooks: in the footsteps of Thomas Kuhn. Am J Public Health 1999; 89: 1162-1165.

12. Susser E, Bresnahan M. Origins of epidemiology. Ann NY Acad Sci 2001; 954: 6- 18 .

13. Krieger N. Epidemiology and social sciences: toward a critical reengagement in the $21^{\text {st }}$ century. Epidemiol Rev 2000; 22: 155-163. 
14. Kawachi I. Social epidemiology. Soc Sci Med 2002; 54: 1739-1741.

15. Skrabanel P. The emptiness of black box. Epidemiology 1994; 5: 553-555.

16. Krieger N. Theories for social epidemiology in the $21^{\text {st }}$ century: an ecosocial perspective. Int J Epidemiol 2001; 30: 668-677.

17. Susser M, Myer L. Social epidemiology. In: Holland WW, Olsen J, Florey C. The development of modern epidemiology. Personal report from those who were there. Oxford: Oxford University Press; 2007: 207-217.

18. Mackenbach JP. Multilevel ecoepidemiology and parsimony. J Epidemiol Community Health 1998; 52: 608-611.

19. Diez-Roux AV. The study of group-level factors in epidemiology: rethinking variables, study designs, and analytical approaches. Epid Rev 2004; 26: 104-111.

20. McMichael AJ. Prisioners of the proximate: loosening the constraints on epidemiology in an age of change. Am J Epidemiol 1999; 149: 887-897.

21. Paneth N. A conversation with Mervyn Susser. Epidemiology 2003; 14: 748-752.

22. Terris M. The lifestyle approach to prevention: editorial. J Public Health Policy 1980; 1: 5-9.

23. Crawford R. Usted es un peligro para su salud: aspectos ideológicos y políticos de la práctica de culpabilizar a la víctima. En: de la Cuesta C. Salud y enfermedad. Lecturas básicas en sociología de la medicina. Medellín. Editorial Universidad de Antioquia; 1999: 47-77.

24. Rose G. Sick individuals and sick populations. Int J Epidemiol 2001; 30: 427-432.

25. Rose G. Rose's strategy of preventive medicine. Oxford: Oxford University Press; 2008.

26. Pearce N. Traditional epidemiology, modern epidemiology, and public health. Am J Public Health 1996; 86: 678-683.

27. Shy CM. The failure of academic epidemiology: witness for the prosecution. Am J Epidemiol 1997; 145: 479-484.

28. Susser M, Stein Z. Eras in epidemiology. The evolution of ideas. Oxford: Oxford University Press; 2009.

29. Ariza EY, López CM, Martínez O, Arias SA. Ecoepidemiología: el futuro posible de la epidemiología. Rev Fac Nal Salud Publ 2004; 22: 139-145.

30. McMichael AJ. The health of persons, populations, and planets: epidemiology comes full circle. Epidemiology 1995; 6: 633-636.

31. Kuller LH. Invited commentary: circular epidemiology. Am J Epidemiol 1999; 150: $897-903$.

32. Maziak W. The triumph of the null hypothesis: epidemiology in an age of change. Int J Epidemiol 2009; 38: 393-402.

33. Pearce N, Douwes J. Response: time for species-course epidemiology? Int J Epidemiol 2009; 38: 403-410.

34. Vandenbroucke JP. Commentary: Maziak's essay, seen from another angle. Int J Epidemiol 2009; 38: 410-412.

35. Dunn JD. Author's response. The triumph of the null hypothesis: epidemiology in an age of change. Int J Epidemiol 2009; 38: 607.

36. Buchanan AV, Weiss KM, Fullerton SM. Dissecting complex disease: the quest for the Philosopher's Stone? Intern J Epidemiol 2006; 35: 562-571.

37. Ioannidis JPA. Commentary: grading the credibility of molecular evidence for complex diseases. Intern J Epidemiol 2006; 35: 572-577.

38. Ioannidis JPA. Genetic associations: false or true? Trends Mol Med 2003; 9: 135-138.

39. Ioannidis JPA. Microarrays and molecular research: noise discovery? Lancet 2005; 365: 454-456.
40. Hattersley AT, McCarthy MI. What makes a good genetic association study? Lancet 2005; 366: 1315-1323.

41. Michiels S, Koscielny S, Hill C. Prediction of cancer outcome with microarrays a multiple random validation strategy. Lancet $2005 ; 365$ : 488-492.

42. Khoury MJ, McCabe LL, McCabe ERB, Population screening in the age of genomic medicine. $N$ Engl J Med 2003; 348: 50-58.

43. Rockhill B, Kawachi I, Colditz GA. Individual risk prediction and populationwide disease prevention. Epidemiol Rev 2000; 22: 176 -180.

44. Blackburn H. Cardiovascular disease epidemiology. In: Holland WW, Olsen J, Florey C. The development of modern epidemiology. Personal report from those who were there. Oxford: Oxford University Press; 2007: 71-92.

45. Susser M, Susser E. Choosing a future for epidemiology: OO. From black box to Chinese boxes and eco-epidemiology. Am J Public Health 1996; 86: 674-677.

46. Susser M. Does risk factor epidemiology put epidemiology at risk? Peering into the future. J Epidemiol Community Health 1998; 52: 608-611.

47. Kogevinas M. The loss of the population approach puts epidemiology at risk. $J$ Epidemiol Community Health 1998; 52: 615-616.

48. Diez-Roux AV. On genes, individuals, society, and epidemiology. Am J Epidemiol 1998; 148: 1027- 1032.

49. Loomis D, Wing S. Is molecular epidemiology a germ theory for the end of the twentieth century? Intern J Epidemiol 1990; 19: 1-3.

50. Vandenbroucke JP. Is 'The Causes of Cancer' a miasma theory for the end of the twentieth century? Intern J Epidemiol 1988; 15: 708-709.

51. Krieger N, Zierler N. What explains the public's health?-A call for epidemiologic theory. Epidemiology 1996; 7: 107-109.

52. Morris JN. Uses of epidemiology. Br Med J 1955; 395-401.

53. Saiger GL. Ten uses of epidemiology. Can Med Assoc J 1961; 85: 992-995.

54. Rothman KJ, Adami HO, Trichopoulos D. Should the mission of epidemiology include the eradication of poverty? Lancet 1998; 352: 810-813.

55. Savitz DA, Poole C, Miller WC. Reassessing the role of epidemiology in public health. Am J Public Health 1999; 89: 1158-1161.

56. Weed DL, Mink PJ. Roles and responsabilities of epidemiologists. Ann Epidemiol 2002; 12: 67-72.

57. Thacker SB, Duffington. Applied epidemiology for the $21^{\text {st }}$ century. Intern $J$ Epidemiol 2001; 30: 320-325.

58. Pearce N. Epidemiology as a population science. Intern J Epidemiol 1999; 28: S1015-S1018.

59. Smith GD. Reflections on the limitations to epidemiology. J Clin Epidemiol 2001; 54: 325-331.

60. Schwartz S, Susser E, Susser M. A future for epidemiology? Annu Rev Public Health 1999; 20: 15-33.

61. Weed DL. Beyond black box epidemiology. Am J Public Health 1998; 88: 12-14.

62. Susser M, Susser E. Choosing a future for epidemiology: I. Eras and paradigms. Am J Public Health 1996; 86: 668-673.

63. Pearce N, McKinlay. Back to the future in epidemiology and public health: response to dr. Gori. J Clin Epidemiol 1998; 51: 643-646.

64. Krieger N, Alegría M, Alemida-Filho N, Barbosa da Silva J, Barreto ML, Beckfield J, et al. Who, and what, causes health inequities? Reflections on emerging debates from an exploratory Latin American/North American workshop. $J$ Epidemiol Community Health 2010; 64: 747-749.

65. Krieger N. Epidemiology and the people's health. Theory and context. Oxford University Press. New York: Oxford University Press; 2011 